\title{
Study on Chinese-English Translation of Traditional Chinese Medicine Terms in Empresses in the Palace from the Perspective of the Skopos Theory
}

\author{
Zikai Guo \\ Center for Medical Language and Culture Studies, X'an Medical University, Xi'an 710021, China \\ guozikai0704@163.com
}

\begin{abstract}
With the globalization developing and deepened, cross-cultural communication between China and the west has been increasing. Today, in the era of mass media, movies and teleplays are the important media and major ways for the transmission of human culture. In recent years, an increasing number of Chinese teleplays have drawn attention from international community. They have gone global and amazed the foreign audience. The quality of subtitle translation not only affects foreign audiences' understanding of the connotation of teleplays, but also affects the incorporation of a country's culture into the international community. Traditional Chinese medicine has a long history of over two thousand years, which is the brightest pearl in treasure house of Chinese culture. With the external communication of movies and television plays, TCM is stepping in the international community in a more specific way, and TCM translation in movies and teleplays has drawn extensive attention from people from home and abroad. The quality of English translation in traditional Chinese medicine certainly affects its' internationalization. Skopos Theory regards translation as a purposeful cross-cultural communication activity. The Skopos rule, coherence rule and fidelity rule should be followed in translation. The communicative function of film and teleplay subtitle translation determines that the translators should not only consider the differences between two languages, but also consider the purpose and audience expectations, cultural backgrounds, etc. Guided by the three rules of Skopos Theory, appropriate translation strategies should be adopted flexibly. Therefore, Skopos Theory enables a new view for subtitle translation of films and television plays. Guided by the Skopos Theory of Translation function, this paper selects court drama "Empresses in the Palace" and its translation of TCM terms within it as the subject investigated. The contrastive study will be made based on corresponding translation of traditional Chinese medicine terms in Chinese and English in Empresses in the Palace, deconstructing and analyzing its translation so as to summarize the translation strategies and provide a reference for TCM translation.
\end{abstract}

Keywords: Empresses in the Palace; Skopos Theory; traditional Chinese medicine terms; translation strategies.

\section{Introduction}

With globalization stepping up the pace, cross-cultural communication between China and western countries have been booming ever since reform and opening up. More and more foreign experts study Chinese culture, especially TCM. Subtitle translation is significantly different from normal texts. Besides general characteristics, the translators must consider the linguistic and cultural background of target audiences. In China, subtitle translation is not impeccable, and still needs enhancing in terms of inaccuracy of corresponding translations, and a lack of perfect translation system.

Based on six episodes of Empresses in the Palace, this thesis mainly studies the TCM terms in the subtitle translation. There is a great difference between Chinese culture and American culture: translators will be in the face of a great challenge like complicated disease translation, drug translation, and treatment translation. These translations must not only conform to English expression to make target reviewers understandable, but also retain the original characteristics of Chinese culture.

In the framework of Skopos Theory, it holds the view that translation is generally regarded as a purposeful cross-cultural communication activity, and one of the most important factors in determining the purpose of translation is the audiences - the target receivers.

From the perspective of Skopos Theory, this paper presents that the translator applies various strategies to thetranslation of TCM terms. It aims at making further contributions to comprehension 
of Skopos Theory in offering proper strategies to TCM translation, further promoting cross-cultural communication and internationalization of TCM.

\section{Skopos Theory}

Skopostheorie emerged in 1970s in Germany, which applied the concept of Skopos to translation. German Skopos Theory advocates the function of the purpose of translation should be based on to the translation and the translation readers' response to translation, rather than on the basis of traditional theory of translation. They have their own expectations, cultural background for translation and communication needs. Therefore, translation is a text produced for a certain purpose and target audience in the context of the target language. Its core concept was stated as: The main factor is the purpose of the overall translation. Skopos Theory gets rid of traditional translation theory and applies translation studies from a new perspective. The development had undergone four stages. The first stage: Catherine Rice's mode of translation criticism, the second stage: Hans Vermeer proposed the Skopos Theory, the third stage: Jasta Holtz-Mantari's theory of translation behaviors, the fourth stage: Christina Nord added the guiding principle of "function plus loyalty".

The term Skopos came from the Greek word means aim or purpose, and it was introduced in 1970s by Hans Vermeer as a technical term for purpose of translation and the action of translation. The major job on Skopos Theory is ground work for a general theory of translation, a book Vermeer coauthored with Katharina Reiss (Vemeer and Reiss1984). Skopos Theory focuses on above all the purposes of translation, which determines the translation strategies that is to be employed in order to produce a functional adequate result. This result is target text, which Vermeer called the translatum. Therefore, it is Skopos Theory that know about why a source text is to be translated and what function of the target text will be of crucial for translators.

There are three possible kinds of purposes in the field of translation. The general purpose aimed at by the translator in the translation process, the communicative purpose aimed at by the target text in the target situation, and the purpose aimed at by a particular translation strategy or procedure (For example: "To translate literally in order to show the particularities of the source text"). (ptd. In Nord.2001:28). Nevertheless, the term "Skopos" always refers to purpose of the target text. It is receiver, or rather the address which is the main factor that determines the Skopos of target text. Therefore, the prime principle determining all the translation processes is the purpose of the overall translation actions.

In the translation of TCM terms of Empresses in the Palace, the translators should not only convey the text meaning, but also are supposed to be the messengers of cultural communication to promote the internationalization of TCM. Therefore, the translation rules translators should follow are audience-oriented principle and culture transmission. Under the guidance of Skopos Theory, translators are freer to adopt more translation strategies.

\section{Translation Strategies of Empresses in the Palace}

TCM terms are the basis and essence of TCM, which are full of national characteristics and difficulties in translating. It is so complicated, which includes drug translation, disease translation, treatment translation, Yin and Yang, Qi etc. The translation of TCM terms is very important in the international exchange of TCM. Empresses in the Palace is a court play in which the emperor and concubines take a lot of medicated food and tonics for maintenance. At the same time, the imperial concubines fight in the play. All kinds of spices and TCM have also become a popular weapon for concubines. It makes audiences amazed.

This part of Translation Strategies of Empresses in the Palace is classified into four parts. The classification refers to related contents of Differential diagnostics of common symptoms in traditional Chinese Medicine, and Standard of common clinical terms in traditional Chinese Medicine. The current popular ontology is used to supplement the deficiencies of classification. 


\subsection{Strategies in Drug Translation}

White alum [Strategy] literal translation. It refers that not only the content of the original text can be conveyed, but the rhetorical styles and forms are preserved as completely as possible under the permission of language in the process of translation. [Analysis] The translation of this kind of the drug totally follows the rule of word by word translation without any change in rhetoric and form. It not only maintains the original style, but also conveys the meaning of the original text. The translation here is in accord with the expression of the target audiences to make it easier to understand without ambiguity.

Huanyi Incense [Strategy] transliteration. It refers to translating the foreign language with words of target language which is similar in pronunciation. The expression used in transliteration no longer keeps its original meaning, and only preserves its language and writing styles. Generally, it is applicable to the translation of proper noun, ethnic identity and no corresponding words.

\subsection{Strategies in Diseases Translation}

Damage of the spleen, stomach and intestine [Strategy] conversion. It refers to converting parts of speech, sentence patterns, voices of original text to match with the way to expressions, even habits of target language. [Analysis] Translated literally, it should be "the spleen, stomach and intestine are damaged". The statement not only converts the verbal phrase to a nominal one, but its order. It is in accordance with the rule in English expression, which places the importance in the beginning. It both makes the translation brief and natural. Its level is improved drastically, which brings target audiences a kind of enjoyment.

Suffer a shock which has led to her fall ill [Strategy] combination. It is a strategy to combine several short sentences into one long sentence in translation, which is applied to Chinese-English translation in general to make the expression brief and coherent. [Analysis] If translated in Chinese expression, the sentence should be: "suffer a shock", and make her fall ill". The translator combines two shorter phrases into a longer one, which is in accordance with expressions of long sentence in English. It not only makes the sentence brief and coherent, but makes the target audiences easier to understand and accept.

\subsection{Strategies in Treatment Translation}

Recover with treatment [Strategy] omission [Analysis] Translated literally, it' will be like "Recover with careful treatment". The translator omits the unnecessary word "careful" but keeps the statement intact. First of all, it makes the phrase briefer. In addition, it brings great convenience to target viewers in watching and comprehending.

Care for your pregnancy [Strategy] amplification [Analysis] It was said by Doctor Wenshi Chu when ZhenHuan was pregnant. Each word of the English sentence is basically corresponding to that of the Chinese language. It not only implies the meaning of "protection", but "caring", which is an indication of sincere and deep love to ZhenHuan. The translator adopts Pun to show the implication, further making the expression more significant and more vivid. It will be much easier for foreign audience to understand.

\subsection{A Summary of Translation Strategies}

At present, the basic translation strategies in TCM terms mainly include literal translation, free translation, anastrophe, interpretation, omission, simplification, transliteration, formation, context method, repetition, conversion, synthesis, etc. Based on the existing research about the translation strategies of TCM, this thesis adopts literal translation, free translation, transliteration, repetition, addition, anastrophe, conversion, combination, amplification, reorganization, omission, synthesis to analyze TCM translation in Empresses in the Palace.

The great differences between Chinese and English determine the variety of translation strategies of TCM terms. Modern translation theory indicates that the semantic correspondence between the lexical units of the two languages can be divided into three classes: full correspondence, partial correspondence and non- correspondence. When it is full correspondence, literal translation should 
be adopted at first. Literal translation makes it easier for target viewers to understand, which makes the original language turn into linguistic system of target language rapidly. It's suitable for some drug, disease and treatment translation. When it is partial correspondence, flexible translation strategies should be adopted on the basis of specific circumstance. It mainly exists in some disease and anatomy concepts. Actually, context method and calque should be combined to deal with TCM translation. When it is non-correspondence, transliteration, synthesis, addition, omission, amplification etc should be adopted. Translator applies those translation strategies to deal with translation when no counterparts exist in TCM translation.

All in all, in order to further find the best translation counterpart, translator is supposed to adopt various translation strategies to maintain the characteristics of TCM as much as possible on the basis of the content of TCM. Although it looks like a target that is contradictory and difficult to achieve, all the translators should struggle for it lifelong. For the dissemination and globalization of TCM, it is never too late to accelerate our step.

\section{Conclusion}

With globalization developing, the intercultural communication between Chinese and western countries has been booming, the teleplay has set a good example in intercultural communication between China and the west, especially in TCM. It presents various translations of TCM elements in drug translation, disease translation, treatment translation etc in an analyzed and investigative way. It has drawn attention to viewers from domestic and overseas. Although Empresses in the Palace does not achieve too much success on American cultural market, it is an explorative step of TCM to go global.

Under the guidance of Skopos Theory, this paper analyzes the translation of TCM terms systematically and separately. Based on Skopos Theory, the author searches out all the TCM translations from Empresses in the Palace firstly, and analyses typical examples to expound the application of three rules of Skopos Theory in various translation strategies secondly which includes literal translation; free translation; transliteration; addition; omission amplification etc. More attention has been paid to TCM, and better comprehension has been made by foreign viewers. The author tries to provide beneficial references to TCM translation further promoting the cultural transmission of TCM.

The only fly in the ointment is that limitations in TCM translation of Empresses in the Palace result from different social systems, cultural background, manners and customs, and thinking models between China and American. This kind of cultural diversity brings great and potential difficulties to TCM translation. Therefore, more attention should be paid to cultural diversity in the future research, better promoting the understanding of TCM culture all over the world and its internationalization.

All in all, this thesis is just an elementary and explorative translation study in traditional Chinese medicine terms.

\section{Acknowledgements}

This paper is funded by Educational and teaching reform program of Xi'an Medical University (2018JG-48, 2018JG-51); Matching fund program of Xi'an Medical University (2018PT51, 2018PT52); Scientific research program of Department of Education of Shaanxi Province (18JK0650); A Scientific Research Funded by Shaanxi Provincial Health and Family Planning Commission (2016D018).

\section{References}

[1]. Bergmann, J. \& A. Sams. 2012. Flip Your Classroom: Reach Every Student in Every Class Every Day[M]. Washington: International Society for Technology in Education. 
[2]. Clark, R. 1983. Reconsidering Researching on Learning from Media[J]. Review of Education Research, (4): 445-459.

[3]. Lage, M. \& G. Platt. 2000. Inverting the Classroom: A Gateway to Creating an Inclusive Learning Environment[J]. The Journal of Economic Education, (1): 30-43.

[4]. Strayer, J. 2012. How Learning in an Inverted Classroom Influences Cooperation, Innovation and Task Orientation[J]. Learning Environ Res, (15): 171-193. 